\title{
Time-Resolved Observation of Deposition Process of Ultrananocrystalline Diamond/Hydrogenated Amorphous Carbon Composite Films in Pulsed Laser Deposition
}

\author{
Kenji Hanada, ${ }^{1}$ Takashi Nishiyama, ${ }^{2}$ Tsuyoshi Yoshitake, ${ }^{1}$ and Kunihito Nagayama ${ }^{2}$ \\ ${ }^{1}$ Department of Applied Science for Electronics and Materials, Kyushu University, 6-1 Kasuga, \\ Fukuoka 816-8580, Japan \\ ${ }^{2}$ Department of Aeronautics and Astronautics, Kyushu University, 744 Motooka, Nishi-ku, Fukuoka 819-0395, Japan
}

Correspondence should be addressed to Tsuyoshi Yoshitake, yoshitake@asem.kyushu-u.ac.jp

Received 1 November 2008; Accepted 25 November 2008

Recommended by Rakesh Joshi

Optical emission spectroscopy was used to study pulsed laser ablation of graphite in a hydrogen atmosphere wherein ultrananocrystalline diamond (UNCD)/hydrogenated amorphous carbon (a-C:H) composite films were grown on heated substrates. Time-resolved photographs of a plume that expanded from a laser-irradiation spot toward a substrate were taken using a high-speed ICCD camera equipped with narrow-bandpass filters. While the emissions from $\mathrm{C}$ atoms and $\mathrm{C}_{2}$ dimers lasted above the laser-irradiation spot on the target, the emission from $\mathrm{C}^{+}$ions lasted above the substrate surface for approximately 7 microseconds, although the emission lifetime of species is generally approximately 10 nanoseconds. This implies that $\mathrm{C}^{+}$ions actively collided with each other above the substrate surface for such a long time. We believe that the keys to UNCD growth in PLD are the supply of highly energetic carbon species at a high density to the substrate and existence of atomic hydrogen during the growth.

Copyright (C) 2009 Kenji Hanada et al. This is an open access article distributed under the Creative Commons Attribution License, which permits unrestricted use, distribution, and reproduction in any medium, provided the original work is properly cited.

\section{Introduction}

Ultrananocrystalline diamond (UNCD) films are new candidates for hard coatings, semiconducting devices, and microelectromechanical systems (MEMSs). UNCD is obviously distinguished from nanocrystalline diamond (NCD). The grain sizes of NCD and UNCD have been quoted as less than $100 \mathrm{~nm}$ and $2-15 \mathrm{~nm}$, respectively [1]. UNCD films are structurally characterized by the existence of grain boundaries with volume fractions more than $10 \%$ for the total and coexistence of a great proportion of amorphous carbon in the films [1], which induces an extremely large optical absorption [2]. In addition, it is possible for a highly nitrogenated film to possess a high $n$-type conductivity $[3,4]$.

The UNCD films have mostly been fabricated by chemical vapor deposition (CVD) [5]. It is well known that $C_{2}$ dimers have an important role in the growth of UNCD films by CVD [6]. Recently, we have succeeded in growing UNCD films, wherein 5-nm diamond crystallites are embedded in a hydrogenated amorphous carbon (a-C:H) matrix, in a hydrogen atmosphere by pulsed laser deposition (PLD) with a graphite target $[7,8]$. They can be grown at low substrate temperatures and high deposition rates. In addition, while CVD necessitates the pretreatment of substrates on the surface of which diamond crystallites are seeded using diamond powders $[6,9]$, pulsed laser deposition (PLD) does not. In other words, PLD enables not only the growth of UNCD films but also the nucleation of diamond. Since the deposition conditions in PLD are completely different from those in CVD, there must be a difference in the UNCD growth mechanism.

In this paper, we discuss the UNCD growth mechanism in PLD on the basis of previous reports and the experimental results of this study. In this study, we deposited UNCD films, and by using a high-speed intensified charge-coupled (ICCD) camera, we observed the ablation process of graphite under the deposition conditions of these films. Further, we evaluated the structure of the deposited films. 


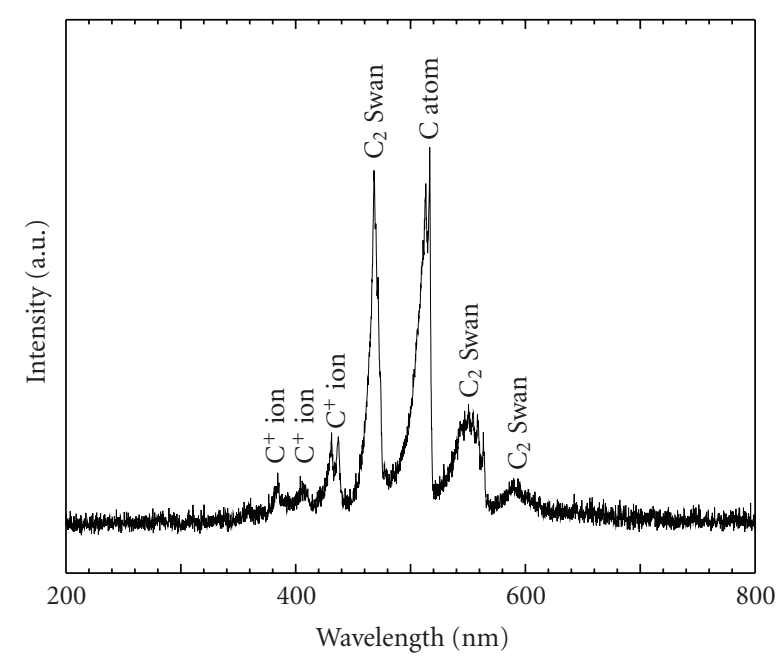

Figure 1: Optical emission spectrum of the entire plume.

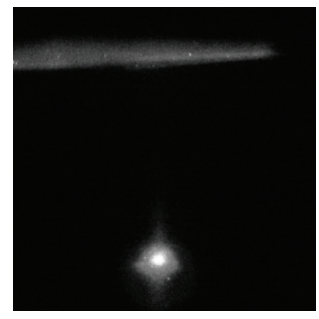

$100 \mathrm{~ns}$

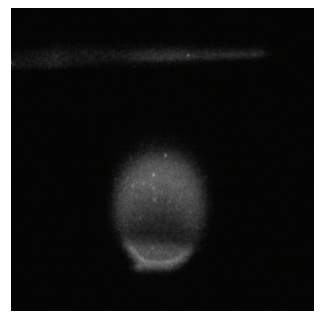

$400 \mathrm{~ns}$

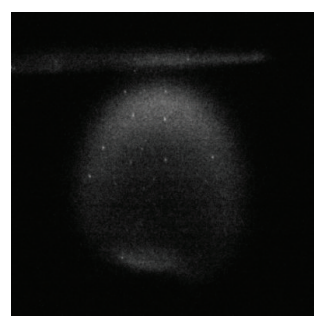

$1 \mu \mathrm{s}$

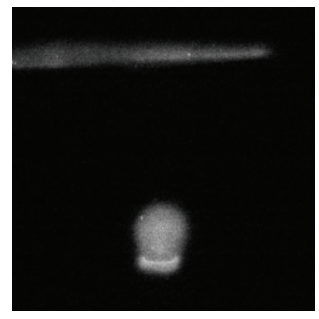

$200 \mathrm{~ns}$

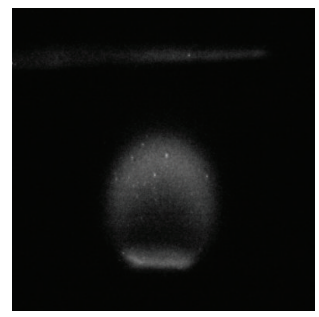

$500 \mathrm{~ns}$

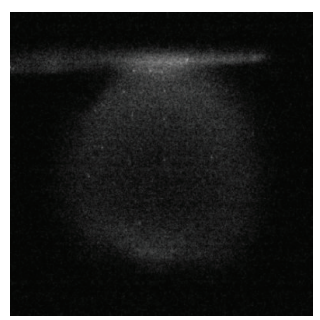

$1.5 \mu \mathrm{s}$

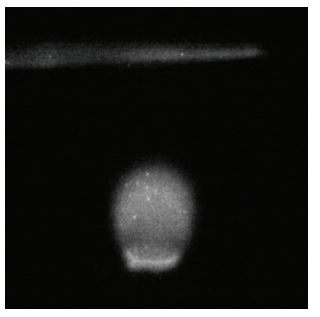

$300 \mathrm{~ns}$

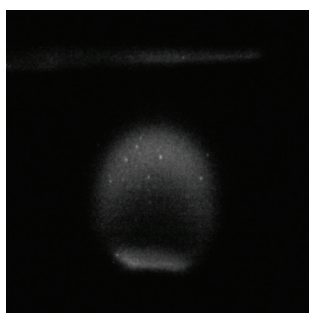

$600 \mathrm{~ns}$

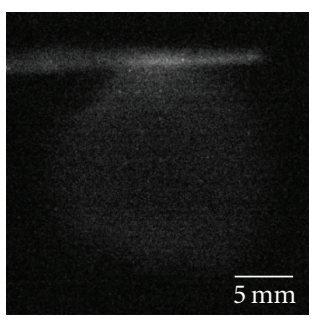

$2 \mu \mathrm{s}$

FIGURE 2: Time-resolved photographs taken without using a filter.

\section{Experimental Section}

Optical emission spectroscopy was used to study the laser ablation process. Observations were made under the optimum deposition condition of the UNCD/a-C:H films. The film preparation conditions have been detailed in our previous papers $[2,10]$. Optical emission spectra of a plume were measured in the wavelength region of 200 to $800 \mathrm{~nm}$ by using a multichannel spectrometer (Ocean Photonics, USB4000). Time-resolved plume images were recorded using an ICCD camera system (C7972-11) capable of recording 12-bit images and equipped with 


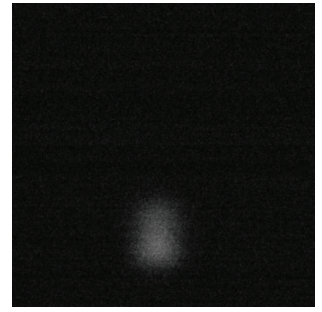

$200 \mathrm{~ns}$

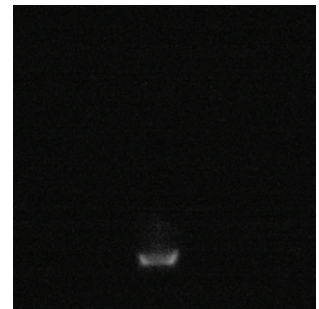

$200 \mathrm{~ns}$

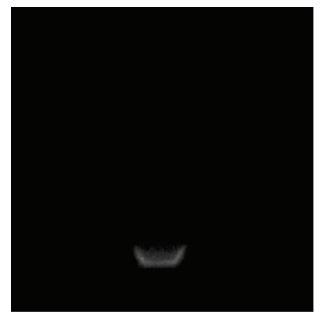

$200 \mathrm{~ns}$

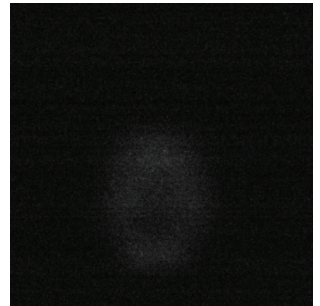

400 ns

(a)

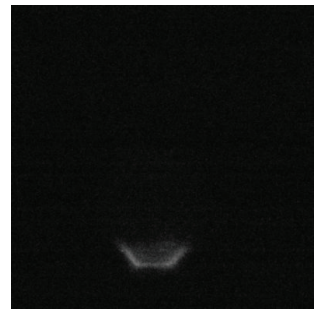

400 ns

(b)

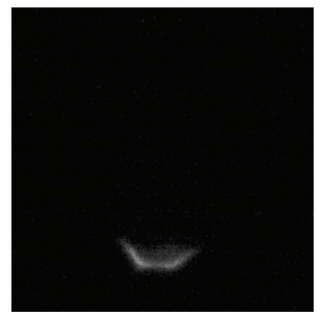

400 ns

(c)

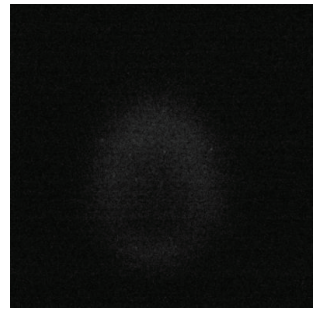

$600 \mathrm{~ns}$

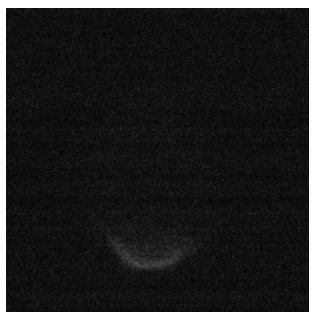

600 ns

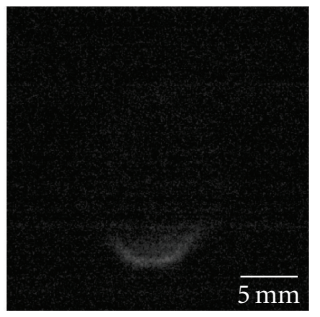

600 ns

Figure 3: Time-resolved photographs captured using (a) 394-nm $\left(\mathrm{C}^{+}\right.$ion), (b) 505-nm (C atom), and (c) 515-nm ( $\mathrm{C}_{2}$ dimer) bandpass filters.

an 18-mm diameter image intensifier and a $1024 \times 1024$ pixel CCD array. In order to observe the behavior of $\mathrm{C}^{+}$ions, $\mathrm{C}$ atoms, and $\mathrm{C}_{2}$ dimers separately, narrowbandpass filters transparent to the wavelengths of 394, 505, and $515 \mathrm{~nm}$ were placed between the vacuum chamber and the lens. The wavelengths of 394, 505, and $515 \mathrm{~nm}$ correspond to those of the representative emission lines of $\mathrm{C}^{+}$ions, $\mathrm{C}$ atoms, and $\mathrm{C}_{2}$ dimers, respectively. The images were taken from laser-irradiation until the plume disappeared, at an interval of $100 \mathrm{~nm}$ and an exposure time of $20 \mathrm{~ns}$.

\section{Results and Discussion}

A typical optical emission spectrum of the plume is shown in Figure 1. The spectrum is continuous at wavelengths between 300 and $700 \mathrm{~nm}$, and the intense peaks that originate from $\mathrm{C}_{2}$ Swan bands, $\mathrm{C}$ atoms, and $\mathrm{C}^{+}$ions were broadened. This is evidently due to Bremsstrahlung radiation. High-density plasma that is similar to previously reported ablation plumes was formed [11]. The emission intensity was markedly large in the area surrounding the laser irradiation spot on the target. The spectrum shown in Figure 1 predominantly comprises the emissions from that area.

Figure 2 shows the time-resolved plume images captured using the high-speed ICCD camera without a bandpass filter. The plume gradually expanded with time. It is well known that a plume expands behind a traveling shock wave in this pressure range [11]. Although the emission lifetimes of species are approximately 10 nanoseconds in general [11], the emission lasted up to $20 \mu \mathrm{s}$. This indicates that the plume has a sufficiently high density for the species to collide with each other. The plume front area located behind the shock wave markedly emitted since the species ejected from the target caught up with the shock wave and gathered inside this area [12]. The front edge of the plume reached the substrate, which was $15 \mathrm{~mm}$ away from the target, at delay times between 1.0 and $1.5 \mu \mathrm{s}$. After that, the emission above the substrate surface further lasted for several microseconds. This evidently implies that high densities of species are present above the substrate surface for such a long time. This situation must have a significant role in UNCD growth. 


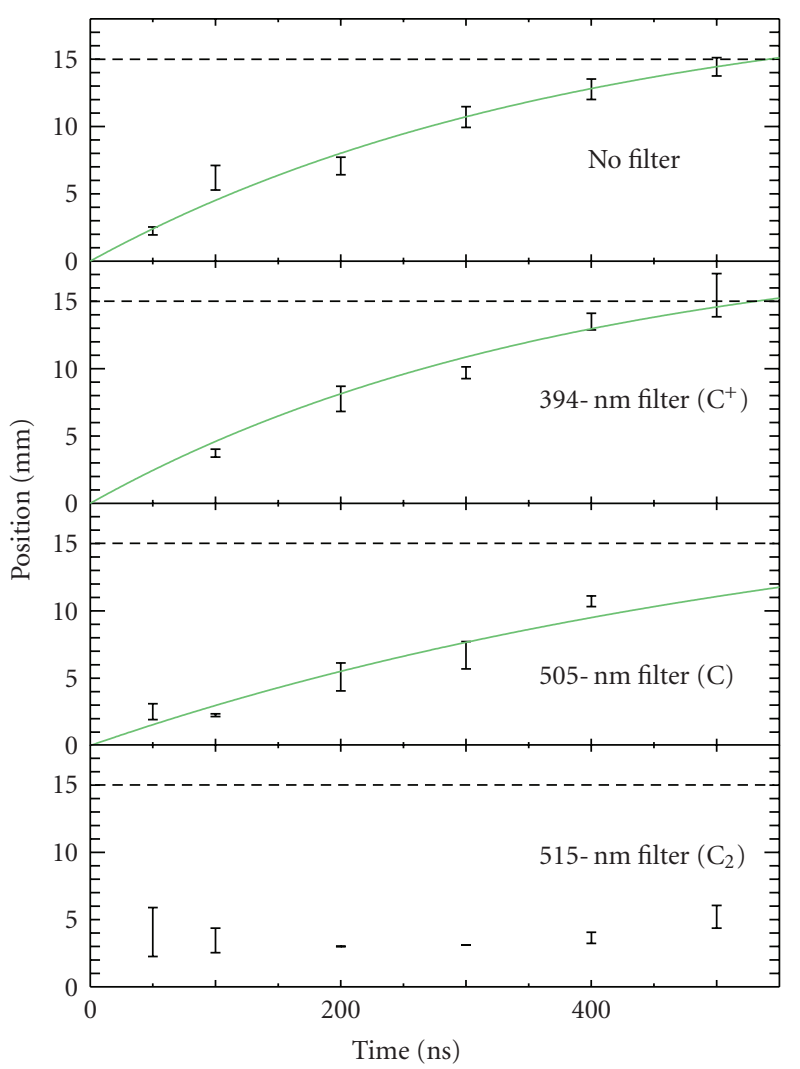

FIGURE 4: Change in the front-edge position of the plume with time. Here, the laser-irradiation spot is defined as the origin.

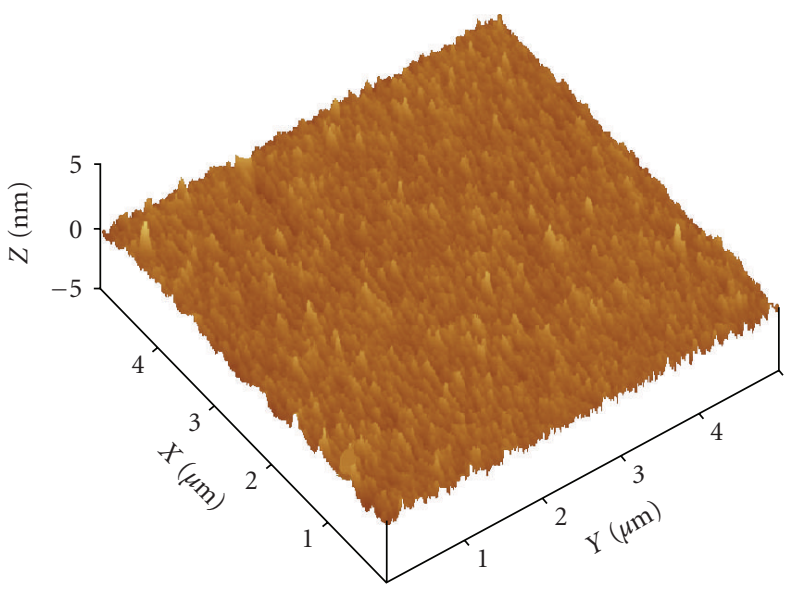

FIGURE 5: AFM image of the UNCD/a-C:H film deposited on Si.

In order to observe the behavior of $\mathrm{C}^{+}$ions, $\mathrm{C}$ atoms, and $\mathrm{C}_{2}$ dimers in the plume separately, time-resolved images were taken using narrow-bandpass filters. Typical time-resolved images for $\mathrm{C}^{+}$ions, $\mathrm{C}$ atoms, and $\mathrm{C}_{2}$ dimers are shown in Figures 3(a), 3(b), and 3(c), respectively. While the emissions from $\mathrm{C}$ atoms and $\mathrm{C}_{2}$ dimers lasted around the target, the emission from $\mathrm{C}^{+}$ions was observed for $7 \mu$ s above the substrate surface. The emission observed above the substrate surface in the images captured without using the filters is evidently attributable to $\mathrm{C}^{+}$ions. It was evidently shown that high-energy $\mathrm{C}^{+}$ions are present in high density above the substrate surface.

In Figure 4, the plume's front-edge position from the target surface is plotted as a function of time. The frontedge positions were carefully determined from the images that were digitally analyzed using image processing software. The plume expansion was fitted with the drag model [12], and the expansion velocity at the substrate was estimated to be $14 \mathrm{~km} / \mathrm{s}$ for $\mathrm{C}^{+}$ions. For the other species, the expansion velocities were impossible to be estimated since their emissions disappeared from the front edge of the plume.

On the basis of previous reports and the experimental results of this study, the UNCD growth mechanism can be considered to be as follows. (i) The root-mean-square roughness of the film surface was estimated to be $0.6 \mathrm{~nm}$ by using atomic force microscopy (AFM), as shown in Figure 5 . This value is extremely small as compared to that of the UNCD films prepared by CVD [13]. This implies that UNCD crystallites are formed at a subsurface rather than the film surface. It has been reported that diamond nucleation occurs when highly energetic $\mathrm{C}^{+}$ions are implanted into amorphous carbon [14]. This does not contradict the fact that the pretreatment of substrates is not required for PLD, while it is indispensable for CVD. We believe that the supply of highly energetic $\mathrm{C}^{+}$ions at a high density to the substrate has an important role in UNCD growth in PLD. Here we must emphasize that this does not have the predominant role on the UNCD growth because the UNCD crystallites do not grow in vacuum wherein the velocities of species must be greater than those in $53.3 \mathrm{~Pa}$ hydrogen atmospheres. Although a 53.3 $\mathrm{Pa}$ hydrogen atmosphere reduces the mean-free-paths and velocities of species, it is indispensable for UNCD growth in PLD. (ii) It has been theoretically predicted that carbon clusters whose dangling bonds are terminated by atomic hydrogen and whose sizes are smaller than $3 \mathrm{~nm}$ will energetically prefer a tetrahedral structure (diamond) to a hexagonal one (graphite) [15]. The UNCD crystallites grown by PLD have an average size of $5 \mathrm{~nm}$. This size is close to the predicted value of $3 \mathrm{~nm}$. Since UNCD growth by PLD requires a hydrogen atmosphere, the existence of atomic hydrogen during UNCD film deposition might contribute to UNCD growth in the mechanism mentioned above. (iii) It has been reported that diamond nucleation is induced under supersaturated conditions [16]. In other words, UNCD crystallites can be formed under supersaturated conditions without pretreating the substrates. Supersaturated conditions might be realized by the presence of high-density $\mathrm{C}^{+}$ions on the substrate surface. The probable mechanisms mentioned above are summarized in Figure 6. We believe that these mechanisms (i), (ii), and (iii) interactively contribute to UNCD growth in PLD. 
Emission from $\mathrm{C}^{+}$ions lasts above the substrate: since the emission lifetime is approximately $10 \mathrm{~ns}$, the prolonged emission indicates that the density of $\mathrm{C}^{+}$ions above the substrate is sufficiently high, causing them to collide with each other.

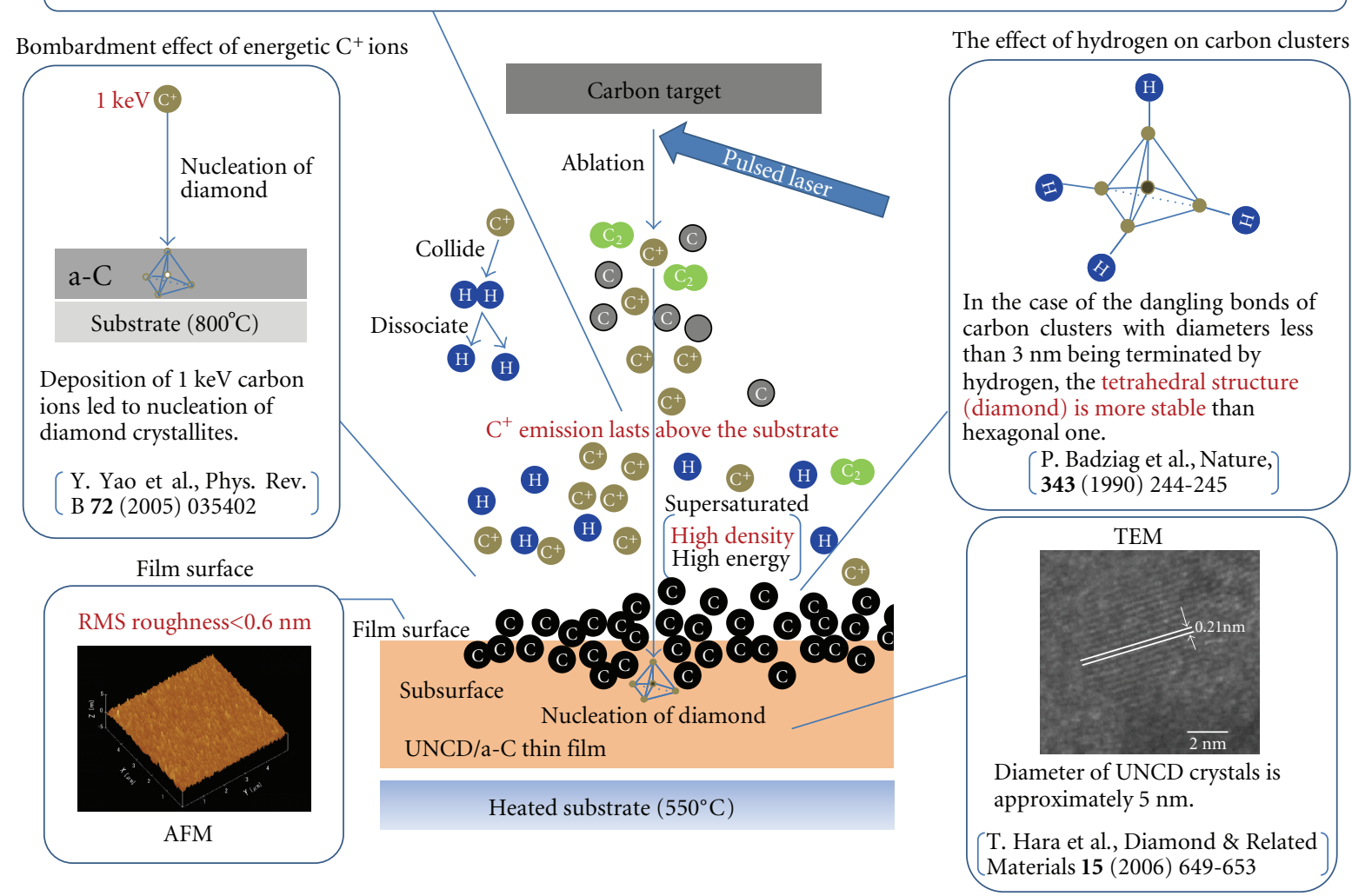

FIGURE 6: Schematic diagram of probable growth mechanisms of UNCD crystallites in the film, based on previous reports and our experimental results.

\section{Conclusions}

Optical emission spectroscopy was used to study pulsed laser ablation of graphite in a hydrogen atmosphere wherein UNCD films were grown. Time-resolved photographs were taken using a high-speed ICCD camera equipped with 394, 505-, and 515-nm filters. Emissions from $\mathrm{C}$ atoms and $\mathrm{C}_{2}$ dimers lasted around the laser-irradiation spot on the target. On the other hand, emission from $\mathrm{C}^{+}$ions lasted above the target surface for approximately 7 microseconds, although the emission lifetime is approximately 10 nanoseconds. This implies that $\mathrm{C}^{+}$ions actively collide with each other above the substrate surface for such a long time. We believe that the keys to UNCD growth in PLD are the supply of energetic carbon species at a high density to the substrate and existence of atomic hydrogen during the growth.

\section{References}

[1] O. A. Williams and M. Nesládek, "Growth and properties of nanocrystalline diamond films," Physica Status Solidi (A), vol. 203, no. 13, pp. 3375-3386, 2006.

[2] T. Yoshitake, A. Nagano, M. Itakura, N. Kuwano, T. Hara, and K. Nagayama, "Spectral absorption properties of ultrananocrystalline diamond/amorphous carbon composite thin films prepared by pulsed laser deposition," Japanese Journal of Applied Physics Part 2, vol. 46, no. 36-40, pp. L936-L938, 2007.

[3] O. A. Williams, S. Curat, J. E. Gerbi, D. M. Gruen, and R. B. Jackman, " $n$-type conductivity in ultrananocrystalline diamond films," Applied Physics Letters, vol. 85, no. 10, pp. 1680-1682, 2004.

[4] P. Achatz, O. A. Williams, P. Bruno, D. M. Gruen, J. A. Garrido, and M. Stutzmann, "Effect of nitrogen on the electronic properties of ultrananocrystalline diamond thin films grown on quartz and diamond substrates," Physical Review B, vol. 74, no. 15, Article ID 155429, 7 pages, 2006.

[5] C. Poppv, W. Kulisch, S. Boycheva, K. Yamamoto, G. Ceccone, and Y. Koga, "Structural investigation of nanocrystalline diamond/amorphous carbon composite films," Diamond and Related Materials, vol. 13, no. 11-12, pp. 2071-2075, 2004.

[6] F. Bénédic, G. Lombardi, K. Hassouni, F. Mohasseb, and A. Gicquel, "Plasma-assisted synthesis: plasma experimental diagnostics and modeling," in Ultrananocrystalline Diamond Synthesis, Properties, and Applications, O. A. Shenderova and D. M. Gruen, Eds., William Andrew, New York, NY, USA, 2006.

[7] T. Yoshitake, T. Hara, T. Fukugawa, et al., "Low-temperature growth of nanocrystalline diamond by reactive pulsed laser deposition under a hydrogen atmosphere," Japanese Journal of Applied Physics, vol. 43, no. 2B, pp. L240-L242, 2004.

[8] T. Hara, T. Yoshitake, T. Fukugawa, et al., "Ultrananocrystalline diamond prepared by pulsed laser deposition," 
Diamond and Related Materials, vol. 15, no. 4-8, pp. 649-653, 2006.

[9] C. Popov, S. Bliznakov, and W. Kulisch, "Influence of the substrate nature on the properties of nanocrystalline diamond films," Diamond and Related Materials, vol. 16, no. 4-7, pp. 740-743, 2007.

[10] A. Nagano, T. Yoshitake, T. Hara, and K. Nagayama, "Optical properties of ultrananocrystalline diamond/amorphous carbon composite films prepared by pulsed laser deposition," Diamond and Related Materials, vol. 17, no. 7-10, pp. 11991202, 2008.

[11] D. B. Geohegan, "Diagnostics and characteristics of laserproduced plasmas," in Pulsed Laser Deposition of Thin Films, G. Hubler and D. B. Chrisey, Eds., John Wiley \& Sons, New York, NY, USA, 1994.

[12] D. B. Geohegan, "Fast intensified-CCD photography of $\mathrm{YBa}_{2} \mathrm{Cu}_{3} \mathrm{O}_{7-x}$ laser ablation in vacuum and ambient oxygen," Applied Physics Letters, vol. 60, no. 22, pp. 2732-2734, 1992.

[13] C. Popov, W. Kulisch, P. N. Gibson, G. Ceccone, and M. Jelinek, "Growth and characterization of nanocrystalline diamond/amorphous carbon composite films prepared by MWCVD," Diamond and Related Materials, vol. 13, no. 4-8, pp. 1371-1376, 2004.

[14] Y. Yao, M. Y. Liao, Th. Köhler, et al., "Diamond nucleation by energetic pure carbon bombardment," Physical Review B, vol. 72, no. 3, Article ID 035402, 5 pages, 2005.

[15] P. Badziag, W. S. Verwoerd, W. P. Ellis, and N. R. Greiner, "Nanometre-sized diamonds are more stable than graphite," Nature, vol. 343, no. 6255, pp. 244-245, 1990.

[16] W. Kulisch, Deposition of Diamond-Like Superhard Materials, chapter 4, Springer, Berlin, Germany, 1999. 

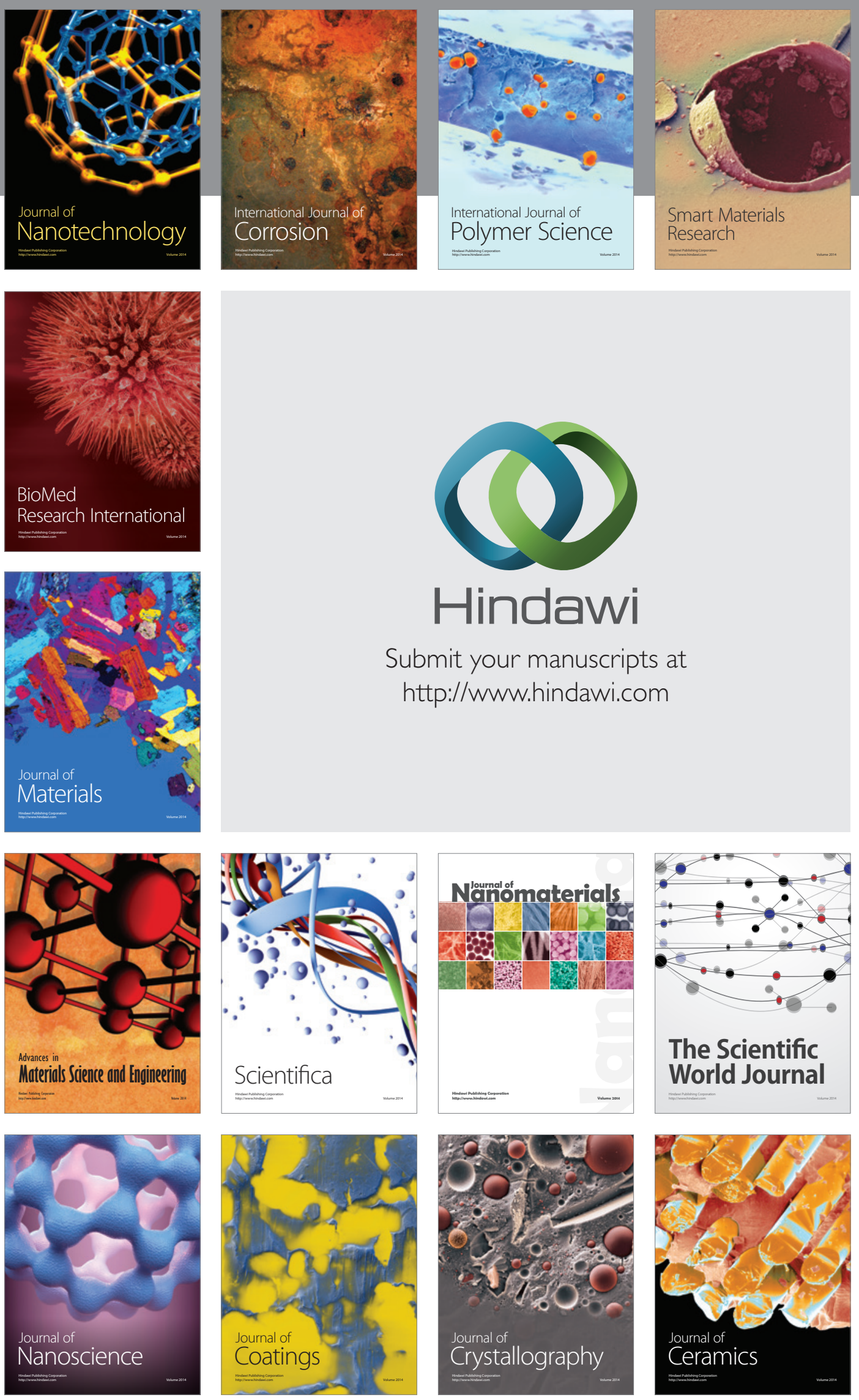

The Scientific World Journal

Submit your manuscripts at

http://www.hindawi.com

\section{World Journal}

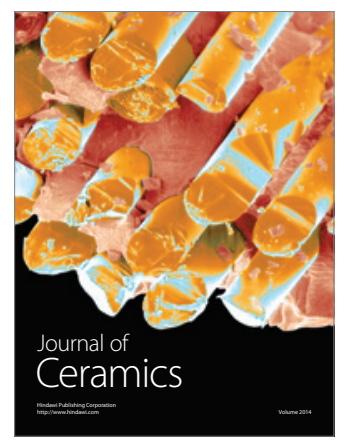

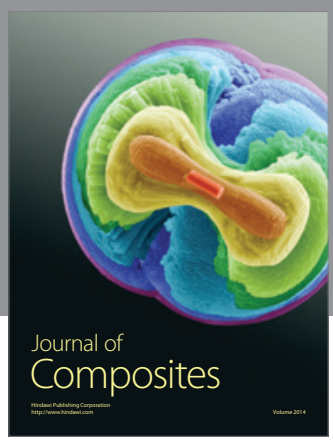
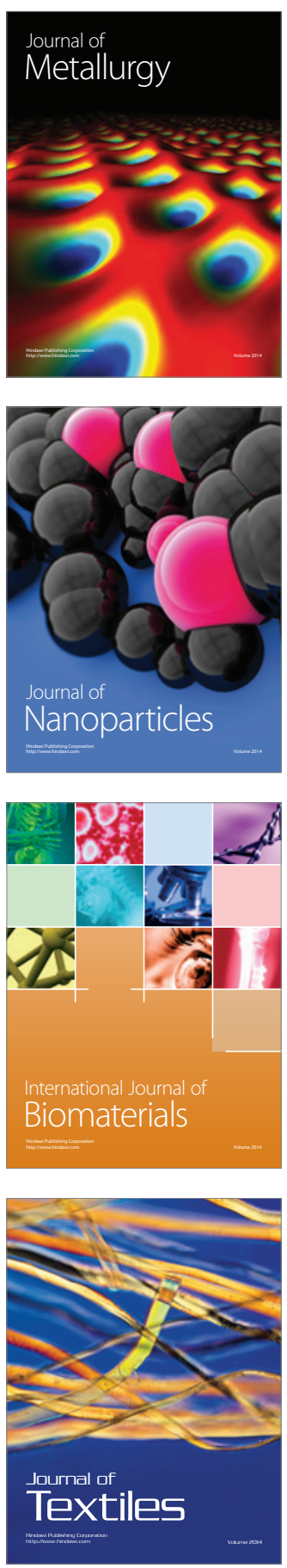